International Journal of Algebra, Vol. 1, 2007, no. 7, 347 - 352

\title{
Parametrization of Torsion Units in $U_{1}\left(\mathbb{Z} D_{5}\right)$
}

\author{
Tevfik Bilgin
}

\author{
Department of Mathematics \\ Faculty of Arts and Sciences \\ Fatih University, Istanbul, Turkey \\ tbilgin@fatih.edu.tr \\ Kamil Ari \\ Department of Mathematics \\ Faculty of Arts and Sciences \\ Yuzuncu Yil University, Van, Turkey \\ kamilari@yahoo.com
}

\begin{abstract}
In this study, a representation of $\mathbb{Z} D_{5}$ is obtained by using a faithful irreducible representation of $2^{\text {nd }}$ degree of $D_{5}$. In the group ring $\mathbb{Z} D_{5}$, all torsion units are expressed in terms of parameters by means of this representation. It is shown that any torsion unit in $U_{1}\left(\mathbb{Z} D_{5}\right)$ can be expressed in terms of four parameters, two of which are free and the others are dependent on free parameters by a quadratic equation.
\end{abstract}

\section{Mathematics Subject Classification: 16U60}

Keywords: Torsion Units, Parametrization

\section{INTRODUCTION}

Let $U_{1}\left(\mathbb{Z} D_{5}\right)$ be normalized unit group of an integral group ring of $D_{5}$. In mid-sixties, Zassenhaus made seemingly very strong conjectures[5]. The first one is

(ZC1) $\quad \gamma \in U_{1}(\mathbb{Z} G),|\gamma|<\infty \Rightarrow \exists g \in G, \quad \alpha \in \mathbb{Q} G$ such that $\gamma=\alpha g \alpha^{-1}$.

This conjecture has been proved by Bhandari and Luthar [1] for metacyclic groups of order $p q$, where $p$ and $q$ are different primes.

Theorem 1.1. Let $G$ be a split extension of a cyclic $p$-group by a cyclic $p^{\prime}$ group with faithful action. Let $u \in U_{1}(\mathbb{Z} G)$ be an element of finite order. Then there exists a $\gamma \in \mathbb{Q} G \in$ such that $u=\gamma^{-1} g \gamma$. 
For a wider class of metacyclic groups, it was given an affirmative answer by Milies and Sehgal [4]. ZC1 was also verified for some metabelian groups by Sehgal and Wiess [6]. Dokuchaev studied on torsion units in integral group ring of nilpotent metabelian groups[2]. In this study, we have given a characterization of torsion units in terms of parameters in the integral group ring of the dihedral group $D_{5}$.

Now, let us write the representation of $\mathbb{Z} D_{5}$ obtained by using faithful irreducible representation of $D_{5}=<a, b: a^{5}=b^{2}=1, b a b^{-1}=a^{-1}>$ (James and Liebeck [3]).

$$
\begin{aligned}
\rho: D_{5} & \rightarrow G L(2, \mathbb{Q}(\omega)) \\
a & \mapsto\left[\begin{array}{cc}
\omega & 0 \\
0 & \bar{\omega}
\end{array}\right] \\
b & \mapsto\left[\begin{array}{ll}
0 & 1 \\
1 & 0
\end{array}\right]
\end{aligned}
$$

where $\omega=e^{\frac{2 \pi i}{5}}$ is the first quintic root of unity and $\bar{\omega}$ is its complex conjugate. By extending this representation $\rho$ of $D_{5}$ over $Z$, we can get the representation $\bar{\rho}$ of $Z D_{5}$ as follows:

$$
\begin{aligned}
\bar{\rho}: \mathbb{Z} D_{5} & \rightarrow \mathbb{Q}(\omega)^{2 x 2} \\
\sum \gamma_{g} g & \rightarrow \sum \gamma_{g} \rho(g)
\end{aligned}
$$

For $\gamma=\alpha_{0}+\alpha_{1} a+\alpha_{2} a^{2}+\alpha_{3} a^{3}+\alpha_{4} a^{4}+\beta_{0} b+\beta_{1} b a+\beta_{2} b a^{2}+\beta_{3} b a^{3}+\beta_{4} b a^{4} \in \mathbb{Z} D_{5}$, Let us denote $\alpha=\sum \alpha_{i} \omega^{i}$ and $\beta=\sum \beta_{i} \omega^{i}$ then the representation of $\gamma$ can be shortened as follows:

$$
\bar{\rho}(\gamma)=\left[\begin{array}{ll}
\alpha & \bar{\beta} \\
\beta & \bar{\alpha}
\end{array}\right]
$$

Now, let us recall some basic results which are used to characterize the torsion units of $U_{1}\left(\mathbb{Z} D_{5}\right)$.

Lemma 1.2. Let $G$ be a metacyclic group and $N=G^{\prime}$, its commutator subgroup. Consider the following natural ring homomorphism:

$$
\begin{gathered}
\varphi: \mathbb{Q} G \rightarrow \mathbb{Q}(G / N) \\
\sum \gamma_{g} g \rightarrow \sum \gamma_{g} g N .
\end{gathered}
$$

If $\gamma \in U_{1}(\mathbb{Z} G)$ is a rational conjugate to for some $g \in G$, then $\varphi(\gamma)=g N$.

Proof. Let $\gamma \in U_{1}(\mathbb{Z} G)$ be a torsion unit. If $\gamma$ is a rational conjugate to $g$ for some $g \in G$, then we write, $\gamma=\alpha g \alpha^{-1}$ for some $\alpha \in \mathbb{Q} G$. Since $G / N$ is abelian and $\varphi$ is the natural ring homomorphism, we have

$$
\varphi(\gamma)=\varphi\left(\alpha g \alpha^{-1}\right)=\varphi(\alpha) \varphi(g) \varphi(\alpha)^{-1}=\varphi(g)=g N
$$


Lemma 1.3. Let $\bar{\rho}$ be the representation of $\mathbb{Z} G$ obtained by linear extension of a representation $\rho$ of finite degree of a group $G$. If $\gamma \in U_{1}(\mathbb{Z} G)$ is a rational conjugate to $g$, for some $g \in G$ then $|\bar{\rho}(\gamma)|=|\rho(g)|$.

Lemma 1.4. Let $\bar{\rho}$ be the representation of $\mathbb{Z} G$ obtained by linear extension of a representation $\rho$ of finite degree of a group $G$. If $\gamma \in U_{1}(\mathbb{Z} G)$ is a rational conjugate to $g$, for some $g \in G$ then $\operatorname{tr} \bar{\rho}(\gamma)=\operatorname{tr} \rho(g)$.

Proposition 1.5 (Berman-Higman[5]). Let $G$ be a finite group. Suppose $\gamma \in U(\mathbb{Z} G)$ is a torsion unit, namely, $\gamma^{n}=1$ for some natural number $n$. If $\gamma_{e} \neq 0$ then $\gamma= \pm 1$.

Corollary 1.6. If $\gamma \in U_{1}(\mathbb{Z} G)$ is a rational conjugate to $g \in \mathcal{Z}(G)$, then $\gamma$ $=g$. That is, any torsion unit which is rational conjugate to an element in the center of a group is trivial.

\section{Parametrization of TORsion Units}

By Theorem 1.1, we can say that any torsion unit of $U_{1}\left(\mathbb{Z} D_{5}\right)$ must be conjugate to one of the conjugate classes of $D_{5}=\{e\} \cup\left\{a, a^{4}\right\} \cup\left\{a^{2}, a^{3}\right\} \cup$ $\left\{b, b a, b a^{2}, b a^{3}, b a^{4}\right\}$. By Corollary 1.6, any torsion unit which is conjugate to $e$ is trivial. As a result, a torsion unit of $U_{1}\left(\mathbb{Z} D_{5}\right)$ of order 2 is conjugate to $b$ and a torsion unit of $U_{1}\left(\mathbb{Z} D_{5}\right)$ of order 5 is a conjugate to either $a$ or $a^{2}$. Let us characterize these three different types of torsion units together.

Theorem 2.1. If $\gamma=\alpha_{0}+\alpha_{1} a+\alpha_{2} a^{2}+\alpha_{3} a^{3}+\alpha_{4} a^{4}+\beta_{0} b+\beta_{1} b a+\beta_{2} b a^{2}+$ $\beta_{3} b a^{3}+\beta_{4} b a^{4} \in U_{1}\left(\mathbb{Z} D_{5}\right)$ is a torsion unit and $\gamma \neq 1$. Then among the coefficients of $\gamma$ following relations hold:

i) $\alpha_{1} \alpha_{2}+\alpha_{2} \alpha_{3}+\alpha_{3} \alpha_{4}=\beta_{0} \beta_{1}+\beta_{1} \beta_{2}+\beta_{2} \beta_{3}+\beta_{3} \beta_{4}+\beta_{4} \beta_{0}$

ii) $\alpha_{1} \alpha_{3}+\alpha_{2} \alpha_{4}+\alpha_{4} \alpha_{1}=\beta_{0} \beta_{2}+\beta_{1} \beta_{3}+\beta_{2} \beta_{4}+\beta_{3} \beta_{0}+\beta_{4} \beta_{1}$

iii) $\alpha_{0}=0$ and one of the following relations holds. Either

$\alpha_{4}=1-\alpha_{1}, \quad \alpha_{3}=-\alpha_{2}, \quad \beta_{0}+\beta_{1}+\beta_{2}+\beta_{3}+\beta_{4}=0$ or

$\alpha_{4}=-\alpha_{1}, \quad \alpha_{3}=1-\alpha_{2}, \quad \beta_{0}+\beta_{1}+\beta_{2}+\beta_{3}+\beta_{4}=0$ or,

$\alpha_{4}=-\alpha_{1}, \quad \alpha_{3}=-\alpha_{2}, \quad \beta_{0}+\beta_{1}+\beta_{2}+\beta_{3}+\beta_{4}=1$.

Proof. Let $\gamma \in U_{1}\left(\mathbb{Z} D_{5}\right)$ be a torsion unit different from 1. By Lemma 1.2, Since $N=D_{5}^{\prime}=<a>$, we have

$$
\varphi(\gamma)=N \text { or } b N \Rightarrow\left\{\begin{array}{l}
\sum_{i=0}^{4} \alpha_{i}=0, \sum_{i=0}^{4} \beta_{i}=1 \text { or } \\
\sum_{i=0}^{4} \alpha_{i}=1, \sum_{i=0}^{4} \beta_{i}=0 .
\end{array}\right.
$$

If we choose $\delta=\sum_{i=0}^{4} \alpha_{i}$, then we write $1-\delta=\sum_{i=0}^{4} \beta_{i}$. since $\delta$ and $1-\delta$ are idempotents, we have

$$
\delta=\left(\sum \alpha_{i}\right)^{2}=\sum \alpha_{i}^{2}+2 \sum \alpha_{j} \alpha_{k} \quad(0 \leq i \leq 4,0 \leq j<k \leq 4) .
$$


By taking $\alpha_{5}=\alpha_{0}$ and $\alpha_{6}=\alpha_{1}$, we get the square of the norm of $\alpha$ as follows

$$
\|\alpha\|^{2}=\left(\sum_{i=0}^{4} \alpha_{i} \omega^{i}\right)\left(\sum_{j=0}^{4} \alpha_{j} \bar{\omega}^{j}\right)=\sum_{i=0}^{4} \alpha_{i}^{2}+(\omega+\bar{\omega}) \sum_{i=0}^{4} \alpha_{i} \alpha_{i+1}+\left(\omega^{2}+\bar{\omega}^{2}\right) \sum_{i=0}^{4} \alpha_{i} \alpha_{i+2}
$$

or equivalently, for $0 \leq i \leq 4$ and $0 \leq j<k \leq 4$,we write

$$
2\|\alpha\|^{2}=2 \sum \alpha_{i}^{2}-\sum \alpha_{j} \alpha_{k}-\sqrt{5} \sum(-1)^{j-k} \alpha_{j} \alpha_{k} .
$$

Similarly, let us compute the square of the norm of $\beta$ :

$$
(1-\delta)=\left(\sum \beta_{i}\right)^{2}=\sum \beta_{i}^{2}+2 \sum \beta_{j} \beta_{k}(0 \leq i \leq 4,0 \leq j<k \leq 4) .
$$

By assigning $\beta_{5}=\beta_{0}$ and $\beta_{6}=\beta_{1}$, we get the square of the norm of $\beta$ as follows:

$$
\|\beta\|^{2}=\sum_{i=0}^{4} \beta_{i}^{2}+(\omega+\bar{\omega}) \sum_{i=0}^{4} \beta_{i} \beta_{i+1}+\left(\omega^{2}+\bar{\omega}^{2}\right) \sum_{i=0}^{4} \beta_{i} \beta_{i+2}
$$

or equivalently, for $0 \leq i \leq 4$ and $0 \leq j<k \leq 4$, we write

$$
2\|\beta\|^{2}=2 \sum \beta_{i}^{2}-\sum \beta_{j} \beta_{k}-\sqrt{5} \sum(-1)^{j-k} \beta_{j} \beta_{k} .
$$

Now let us consider Lemma 1.3

$$
|\bar{\rho}(\gamma)|=\|\alpha\|^{2}-\|\beta\|^{2}=\left(\sum_{i=0}^{4} \alpha_{i} \omega^{i}\right)\left(\sum_{j=0}^{4} \alpha_{j} \bar{\omega}^{j}\right)-\left(\sum_{i=0}^{4} \beta_{i} \omega^{i}\right)\left(\sum_{j=0}^{4} \beta_{j} \bar{\omega}^{j}\right) .
$$

By using identities in (2.3) and (2.5), for $0 \leq i \leq 4$ and $0 \leq j<k \leq 4$, we get

$$
2|\bar{\rho}(\gamma)|=2 \sum\left(\alpha_{i}^{2}-\beta_{i}^{2}\right)-\sum\left(\alpha_{j} \alpha_{k}-\beta_{j} \beta_{k}\right)-\sqrt{5} \sum(-1)^{j-k}\left(\alpha_{j} \alpha_{k}-\beta_{j} \beta_{k}\right) .
$$

On the other hand, by identities in (2.2) and (2.4), for $0 \leq i \leq 4$ and $0 \leq j<$ $k \leq 4$, we obtain

$$
2(|\bar{\rho}(\gamma)|+1-2 \delta)=-5 \sum\left(\alpha_{j} \alpha_{k}-\beta_{j} \beta_{k}\right)-\sqrt{5} \sum(-1)^{j-k}\left(\alpha_{j} \alpha_{k}-\beta_{j} \beta_{k}\right) .
$$

Now let us check all possible values of $2(|\bar{\rho}(\gamma)|+1-2 \delta)$. If $\gamma$ is a rational conjugate to $a$ or $a^{2}$ then by Lemma 1.3 and Lemma 1.4, we have $|\bar{\rho}(\gamma)|=$ $1=\delta$. If $\gamma$ is a rational conjugate to $b$ then by the same reason, we have $|\bar{\rho}(\gamma)|=-1$ and $\delta=0$. In both cases, $|\bar{\rho}(\gamma)|+1-2 \delta=0$. Hence, for $0 \leq i \leq 4$ and $0 \leq j<k \leq 4$ we get

$$
\sum \alpha_{j} \alpha_{k}=\sum \beta_{j} \beta_{k} \text { and } \sum(-1)^{j-k} \alpha_{j} \alpha_{k}=\sum(-1)^{j-k} \beta_{j} \beta_{k}
$$


or equivalently by taking $\alpha_{5}=\alpha_{0}, \alpha_{6}=\alpha_{1}, \beta_{5}=\beta_{0}$ and $\beta_{6}=\beta_{1}$, we write

$$
\sum_{i=0}^{4} \alpha_{i} \alpha_{i+1}=\sum_{i=0}^{4} \beta_{i} \beta_{i+1} \text { and } \sum_{i=0}^{4} \alpha_{i} \alpha_{i+2}=\sum_{i=0}^{4} \beta_{i} \beta_{i+2} .
$$

Since $\gamma$ is a torsion unit different from 1, by Proposition 1.5 we have $\alpha_{0}=0$. Therefore, identities in (2.8) can be written as follows:

$$
\begin{aligned}
& \alpha_{1} \alpha_{2}+\alpha_{2} \alpha_{3}+\alpha_{3} \alpha_{4}=\beta_{0} \beta_{1}+\beta_{1} \beta_{2}+\beta_{2} \beta_{3}+\beta_{3} \beta_{4}+\beta_{4} \beta_{0} \\
& \alpha_{1} \alpha_{3}+\alpha_{2} \alpha_{4}+\alpha_{4} \alpha_{1}=\beta_{0} \beta_{2}+\beta_{1} \beta_{3}+\beta_{2} \beta_{4}+\beta_{3} \beta_{0}+\beta_{4} \beta_{1} .
\end{aligned}
$$

On the other hand by Lemma 1.3 and Lemma 1.4, If $\gamma$ is a rational conjugate to $a$ then

$$
\alpha_{4}=1-\alpha_{1}, \alpha_{3}=-\alpha_{2}, \beta_{0}+\beta_{1}+\beta_{2}+\beta_{3}+\beta_{4}=0
$$

If $\gamma$ is a rational conjugate to $a^{2}$ then

$$
\alpha_{4}=-\alpha_{1}, \alpha_{3}=1-\alpha_{2}, \beta_{0}+\beta_{1}+\beta_{2}+\beta_{3}+\beta_{4}=0
$$

If $\gamma$ is a rational conjugate to $b$ then

$$
\alpha_{4}=-\alpha_{1}, \alpha_{3}=-\alpha_{2}, \beta_{0}+\beta_{1}+\beta_{2}+\beta_{3}+\beta_{4}=1 .
$$

Remark 2.2. Let $\gamma \in U_{1}\left(\mathbb{Z} D_{5}\right)$ be a torsion unit different from 1 . Then we write $3^{\text {rd }}$ relation in Theorem(2.1) as follows:

$$
\alpha_{4}=\delta_{1}-\alpha_{1}, \alpha_{3}=\delta_{2}-\alpha_{2}, \beta_{0}+\beta_{1}+\beta_{2}+\beta_{3}+\beta_{4}=1-\left(\delta_{1}+\delta_{2}\right) .
$$

where $\delta_{1}$ and $\delta_{2}$ determined by $\operatorname{tr} \bar{\rho}(\gamma)=\delta_{1}(\omega+\varpi)+\delta_{2}\left(\omega^{2}+\varpi^{2}\right)$.

Proposition 2.3. If the equation $v^{2}-u v-u^{2}= \pm 1$ holds for $(v, u)$ integer pairs then, it is the same as to say that the equation $v^{2}-u v-u^{2}=1$ holds for $(v, u)$ integer pairs.

Proof. If $(v, u)$ integer pairs hold $v^{2}-u v-u^{2}=1$ then we have

$$
-1=-v^{2}+u v+u^{2}=\left(-u^{2}\right)-(-u) v-v^{2} .
$$

So, $(-u, v)$ pairs hold the negative sign of the same equation.

Theorem 2.4. A torsion unit in $U_{1}\left(\mathbb{Z} D_{5}\right)$ different from 1 can be written as 4 parameters, two of which are free and the others depend on free parameters by a quadratic equation.

Proof. Let $\gamma=\sum_{i=0}^{4} \alpha_{i} a^{i}+\beta_{i} b a^{i} \in U_{1}\left(\mathbb{Z} D_{5}\right)$ be a torsion unit different from 1 . By substituting $\beta_{1}-\alpha_{1}=p, \beta_{2}-\alpha_{2}=q, \beta_{3}-\alpha_{3}=r, \beta_{4}-\alpha_{4}=s$, the equations in Theorem 2.1 we get

$$
1-2\left(\delta_{1}+\delta_{2}\right)-p-q-r-s=\beta_{0} \text { and }
$$


$\beta_{0}\left(p+s+\delta_{1}\right)+r \delta_{1}+(q+s) \delta_{2}+p q+q r+r s=\alpha_{1}(r-q)+\alpha_{2}(-p+q-r+s)$,

$\beta_{0}\left(p+s+\delta_{2}\right)+(p+q) \delta_{1}+p \delta_{2}+p r+q s+p s=\alpha_{1}(-r+q+p-s)+\alpha_{2}(p-s)$.

Now let us substitute once more as $r-q=u$ and $s-p=v$ then we write

$$
\left[\begin{array}{cc}
u & v-u \\
-u-v & -v
\end{array}\right]\left[\begin{array}{l}
\alpha_{1} \\
\alpha_{2}
\end{array}\right]=\left[\begin{array}{c}
\beta_{0}\left(p+s+\delta_{1}\right)+r \delta_{1}+(q+s) \delta_{2}+p q+q r+r s \\
\beta_{0}\left(p+s+\delta_{2}\right)+(p+q) \delta_{1}+p \delta_{2}+p r+q s+p s
\end{array}\right]
$$

Since the square matrix above is an unimodular matrix $v^{2}-u v-u^{2}= \pm 1$. But by Remark 2.2 we can write $v^{2}-u v-u^{2}=1$ for integer pairs $(v, u)$. then we can get the following solution :

$$
\begin{gathered}
\alpha_{1}=-v\left[\beta_{0}-\beta_{0}^{2}+(p+q+r) \delta_{1}+(p+q+s) \delta_{2}+p q+p r+p s+q r+q s+r s\right] \\
+u\left[\beta_{0}\left(q+r+\delta_{2}\right)+(p+q) \delta_{1}+s \delta_{2}+p r+q s+r s\right], \\
\alpha_{2}=u\left[\beta_{0}-\beta_{0}^{2}+(p+q+r) \delta_{1}+(p+q+s) \delta_{2}+p q+p r+p s+q r+q s+r s\right] \\
+v\left[\beta_{0}\left(p+s+\delta_{1}\right)+r \delta_{1}+(q+s) \delta_{2}+p q+q r+r s\right] .
\end{gathered}
$$

Consequently, all coefficient of a torsion unit different from 1 can be written in terms of four parameters, where $p, q \in \mathbb{Z}$ free parameters and $u, v \in \mathbb{Z}$ parameters depend on each other by a quadratic equation $v^{2}-u v-u^{2}= \pm 1$. If we assign $\lambda=\operatorname{sgn}\left(v^{2}-u v-u^{2}\right)$ then the other coefficient are as follows :

$\alpha_{0}=0, \quad \alpha_{3}=\delta_{2}-\lambda \alpha_{2}, \quad \alpha_{4}=\delta_{1}-\lambda \alpha_{1}, \quad \beta_{0}=1-2\left(\delta_{1}+\delta_{2}\right)-p-q-r-s$, $\beta_{1}=p+\lambda \alpha_{1}, \quad \beta_{2}=q+\lambda \alpha_{2}, \quad \beta_{3}=r-\alpha_{2}+\delta_{2}, \beta_{4}=s-\alpha_{1}+\delta_{1}$.

Corollary 2.5. any torsion unit in $U_{1}\left(\mathbb{Z} D_{5}\right)$ different from 1 can be expressed in terms of four parameters, two of which are free and the others are dependent on free parameters by a quadratic equation.

\section{REFERENCES}

[1] Bhandari, A.K., Luthar, I.S., Torsion units of integral group rings of metacyclic groups, J. Number Theory, 17(1993), 170-183.

[2] Dokuchaev,M., Torsion units in integral group rings of nilpotent metabelian groups, Comm. Algebra 20(1992), 423-435.

[3] James, D.G. and Liebeck, M.W. Representations and characters of groups, Cambridge University press, (1993)

[4] Milies,C.P. and Sehgal,S.K., Torsion units in integral group rings of metacyclic groups, J. Number Theory, 19(1984), 103-114.

[5] Sehgal, S.K., Units in integral group rings, Marcel Dekker, New york, Basel. (1993).

[6] Sehgal, S.K. and Weiss, A., Torsion units in integral group rings of some metabelian groups, J. Algebra 103 (1986), 490-499.

Received: January 26, 2007 